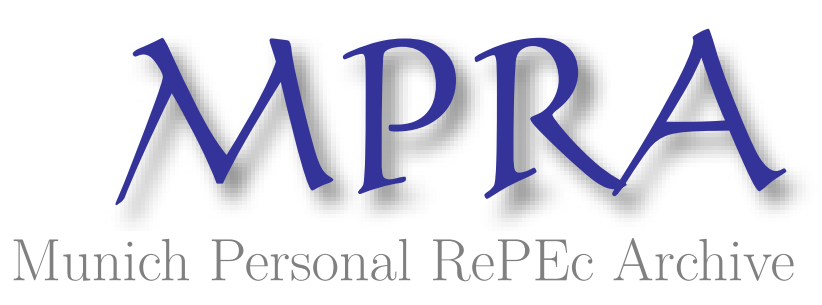

\title{
Regional and Outward Economic Integration in South-East Asia
}

Weber, Enzo

April 2007

Online at https://mpra.ub.uni-muenchen.de/6136/

MPRA Paper No. 6136, posted 07 Dec 2007 00:21 UTC 


\title{
Regional and Outward Economic Integration in South-East Asia ${ }^{1}$
}

\author{
Enzo Weber \\ Universität Mannheim and Freie Universität Berlin \\ Boltzmannstr. 20, 14195 Berlin, Germany \\ eweber@wiwiss.fu-berlin.de \\ phone: +49 30 838-55792 fax: +49 30 838-54142
}

First version: 04/2007

This version: $12 / 2007$

\begin{abstract}
The subject of this paper tackles macroeconomic integration of the South-East Asian countries South Korea, Singapore and Taiwan. Economically, the analysis is based on notions of stochastic long-run convergence and business cycle synchrony in the GDPs. According tests for cointegration and common serial correlation features reveal a high degree of coherence in long-run growth and medium-run fluctuations. This allows extracting a common stochastic growth trend and a common business cycle. Further analysis shows that both of these components are subject to stronger influences from the US than from Japan. Convergence towards these matured economies conspicuously appears since the 1990s.
\end{abstract}

Keywords: Economic Integration, Cointegration, Common Cycles, South-East Asia JEL classification: E32, F15, C32

\footnotetext{
${ }^{1}$ This research was supported by the Deutsche Forschungsgemeinschaft through the CRC 649 "Economic Risk". I am grateful to Jürgen Wolters and Cordelia Thielitz for their help. Of course, all remaining errors are my own.
} 


\section{Introduction}

The last decades of economic development in South-East Asia are marked by vibrant dynamics as the region has witnessed extraordinary growth, which did not go without striking inequality and severe crises. A recent example has been the Asian financial crisis in 1997/98, which occurred in a period of booming economy and deepening interaction between the region's countries. While some nations could make considerable progress, others did far less participate in the dynamic development process. As matter of fact, the members of the main regional organisations, ASEAN and APEC, range from highly industrialised to developing countries, the integration concepts from "open regionalism" to discriminatory trade policies. Thereby, the most successful industrialising economies have soon been called the "Asian Tigers".

From the scientific point of view, it remains an open task to examine the ambiguous character of the South-East Asian economies, even after some work, mainly done on shock identification and income convergence, has shed some light on this topic. For instance, Bayoumi and Eichengreen (1994) find evidence for regional clustering in terms of demand and supply innovations, which bears a certain scope for monetary cooperation. Lee et al. (2004) can establish real long-run convergence of Singapore towards Japan, whereas the remaining ASEAN nations failed in catching up. Surely, the special regional scenery with its varying economic conditions and political historical backgrounds raises several questions:

Most profoundly, does South-East Asia justify using the notion of one economic region? In detail, do the different countries share common macroeconomic characteristics, and which is the nature of transmission effects between them? Seen from a broader perspective, has the regional development led to convergence towards the industrialised world? And at last, which is the importance of foreign economic influences on South-East Asia?

Answering these questions would not only give a more profound insight into the functioning and structure of the world's most dynamic and forward-looking region. On top, it would provide important information for understanding the difficulties in enhancing sustained progress and ensuring macro-stability despite the high vulnerability, which has been a permanent threat in the past. In this context, enhanced cooperation based on deepening integration could bear high potential for stabilisation policies.

This paper approaches the outlined problems in the context of an empirical time series analysis, proceeding on an aggregated macro-level. The examination makes use of recent 
econometric advantages in the field of common features. Namely, the analysis of common stochastic trends and common cyclical dynamics provides adequate means for the given target of finding evidence about stance and course of a macroeconomic convergence: First, relying on the Bernard and Durlauf (1995) concept of income convergence, cointegration is a natural prerequisite for similar growth paths in the long run. Second, synchronous business cycles indicate common grounds mainly in the medium-frequency macroeconomic processes.

The first step of the analysis consists of testing for cointegration and common cycles in the GDPs of South-East Asian countries. Thereby, the exceptionally successful Tigers South Korea, Singapore and Taiwan exemplify the impressive emergence of economic progress. Based on the obtained results, a common growth trend and a common business cycle are filtered out of the data. These series allow interesting insights into long- and medium-run connections to the industrialised world: While considerable impacts from the US become manifest, evidence for convergence strikingly rises in the mid-1990s. The last point gives an answer to the problems in the existing literature of finding support for the time series convergence hypothesis. The proceeding differs from the main existing research directions, which focus for example on the identification and comparison of national supply and demand shocks (Bayoumi and Eichengreen 1994), or cross-section panel convergence; for the latter, see for example Engelbrecht and Kelsen (1999), who characterise the APEC countries as a convergence club.

Preceding the empirical part, section 2 supplies the foundation of the economic concept consisting of theories on convergence, business cycle transmission, growth and optimal currency areas. In the section thereafter, the econometric methods, cointegration, common cycle features and permanent-transitory decomposition, are to be introduced. Section 4 presents the empirical results for the South-East Asian region and its outward relations. Finally, the last section evaluates and summarises the outcome of the econometric investigation.

\section{Economic Foundation}

Before discussing and applying the econometric methods in the next sections, it is worthwhile to briefly consider the economic notion that underlies the following analysis. As has been mentioned in the introduction, the examination aims at grasping the actual stance of regional economic integration in South-East Asia. My concept behind the term 
integration is built on stochastic trending behaviour of the national GDPs in the long run as well as cyclical fluctuations in the short and medium run. The word cycle has not necessarily to be taken literally, but rather in a sense of describing transitory movements in contrast to permanent trending (e.g. Vahid and Engle 1993). Of course, this definition still comprises the original meaning of regular periodical up- and downswings in a macro economy.

The motivation for this concept may be best illustrated by the theory of optimal currency areas. As a core result, it recommends the introduction of a common currency representing a very high stage of integration, if different exchange rates and separated monetary policies have lost their necessity. Above all, this is the case when synchronous cycles facilitate adequate reactions by one common central bank, the foreign exchange is not confronted with a variety of idiosyncratic national shocks and a long-run growth equilibrium between the economies provides preconditions for development in a unified economic system.

Focusing on the latter point, the neoclassical growth theory implies that the steadystate of per capita output does not depend on initial conditions. In detail, diminishing marginal returns are the base for higher growth prospects in countries with lower current income levels, at least theoretically. As a consequence, a common convergence target between different economies is determined in the long run. Only in case of structural microeconomic parameters differing across countries, stable gaps between outputs would persist due to distinct production functions.

In a stochastic framework, Bernard and Durlauf (1996) require for convergence that the gap in outputs between country 1 and 2 is closed while increasing the forecast horizon:

$$
\lim _{k \rightarrow \infty} E_{t}\left(y_{1, t+k}-y_{2, t+k}\right)=0 .
$$

Since this definition rules out persistent shocks to the output gap, it implies that per capita outputs are in pairs cointegrated with the vector $(1,-1)$ (Bernard and Durlauf 1995). In other words, outputs are driven by a single common trend and do therefore exhibit an equilibrium co-movement aligning the steady state levels in the long run. Obviously, this criterion is met by countries, which have already reached this state of integration. Nonetheless, unfinished convergence can be modelled by allowing for a constant and a linear trend in the cointegrating relation. Depending on the direction, deterministic trending could stand for catching-up (Bernard and Durlauf 1996) or for divergence. A constant is to be interpreted as an initial output gap, which could be reduced in case of catching-up, but would otherwise represent a persistent difference. Such a situation might be characterised by the concept of conditional convergence, as explained above. At last, 
under a cointegrating vector other than $(1,-1)$, trend shocks would still be related, even if with different strength.

A similar concept, now for the short-run fluctuations, defines common cycles (Engle and Kozicki 1993) as transitory movements, which are well synchronised among the series and logically cancelled out in a distinct linear combination. A bivariate cofeature vector of $(1,-1)$ would indicate equal per capita business cycle strength. This definition can be weakened by allowing for idiosyncratic components at specific cycle frequencies.

Economic commonalities both in permanent and transitory processes can be the result of identical innovations beyond the bounds of national borders, for example caused by transnational production networks or exposure to world market shocks. But even in absence of such initial cohesion, co-movement can be fostered by transmission mechanisms. According channels discussed in a comprehensive literature shall be shortly presented: Most directly, impulses are propagated, when the demand for foreign goods (in the broadest sense) depends on the domestic economic situation, as it is usually stated in import functions. This export channel (e.g. Canova and Dellas 1993) is of distinct importance in the industrialisation process specifically apparent in South-East Asia. In financial markets, in- and outflow of capital due to real and nominal triggers creates a link between countries; in the focused region, the foreign direct investment channel (e.g. Jansen and Stokman 2004) plays an extraordinarily important role. By the same token, diffusion of technological knowledge, possibly in combination with the aforementioned point, leads to similar shifts in production functions. At last, labour mobility across borders can affect wages, production and consumption. The empirical application following the methodological part shall uncover nature and degree of South-East Asian coherence evolving from such fundamental developments.

\section{Methodological Proceeding}

\subsection{Model Framework}

The basic data generating process in the econometric procedure shall be approximated by a VAR with lag length $q+1$

$$
y_{t}=c_{0}^{*}+c_{1}^{*} t+c_{2}^{*} b_{t}+c_{3} d_{t}+\sum_{i=1}^{q+1} A_{i}^{*} y_{t-i}+u_{t},
$$


where $y_{t}$ contains the $n$ endogenous variables (GDPs), $A_{i}^{*}$ are $n \times n$ coefficient matrices and $u_{t}$ is an $n$-dimensional vector of white noise errors. The deterministic terms are constant, linear trend $(t)$, level breaks $\left(b_{t}\right)$, which might accomodate economic disruptions, and impulse dummies $\left(d_{t}\right)$, which guarantee normality of the residuals.

Given the presence of unit roots in the per capita GDPs, according to Johansen (1995), the commonness of $n-r$ stochastic trends is reflected by a reduced rank of $A^{*}(1)$, with

$A^{*}(L)=I_{n}-\sum_{i=1}^{q+1} A_{i}^{*} L^{i}$. Consequently, one can write $A^{*}(1)=-\alpha \beta^{\prime}$, where $\beta$ spans the space of the $r$ cointegrating vectors, and $\alpha$ contains the corresponding adjustment coefficients. Granger's representation theorem leads to the VECM

$$
\Delta y_{t}=\alpha\left(\beta^{\prime} y_{t-1}+c_{0}+c_{1}(t-1)+c_{2} b_{t-1}\right)+c_{3} d_{t}+\sum_{i=1}^{q} A_{i} \Delta y_{t-i}+u_{t}
$$

with $A_{i}=-\sum_{j=i+1}^{q+1} A_{j}^{*}, i=1, \ldots, q$. This representation assumes that constant, trend and breaks are absorbed in the cointegrating relation. Note that in (3) lagged intervention dummies, which condition the likelihood function in each subsample (defined by the break dates), as in Johansen et al. (2000), are not displayed for simplicity. For illustration, in the bivariate case the real income convergence would imply $\beta^{\prime}=(1,-1)$ in $(3)$.

\subsection{Trend Analysis}

As the basis for long-run growth analysis, the unit root behaviour of (non-breaking) series is checked by ADF tests, with constant, trend and centred seasonal dummies included. The lag length is set following the usual information criteria and autocorrelation tests. Simulated critical values for the null hypothesis of non-stationarity are taken from Davidson and MacKinnon (1993).

Various authors found that the presence of structural breaks distorts the unit root test results, see i.e. Perron (1989). Certainly, there is no doubt that such shifts have occurred in the Asian financial crisis. Here, I follow Saikkonen and Lütkepohl (2002), who propose first estimating the deterministic nuisance parameters and afterwards testing the residuals for non-stationarity. Accordingly, in the first step a GLS regression of the time series on constant, trend, dummies and shift is run. As in the case of the Asian crisis the shift dates are evident, I assume the break points to be known; nevertheless, endogenous break point search does not alter the basic conclusions. In the second step, an ADF-type test on the estimated residuals is performed. For critical values of the t-statistic and additional 
correction terms in the regression see Lanne et al. (2002).

Johansen $(1994,1995)$ provides a test for cointegration in the VECM from (3), Johansen et al. (2000) incorporate structural breaks. Their likelihood ratio (LR) trace test statistic for the null hypothesis of at most $r$ cointegrating relations is given by

$$
\Lambda(r)=-T \sum_{i=r+1}^{n} \log \left(1-\hat{\lambda}_{i}\right)
$$

where $n$ is the number of endogenous variables and $T$ the number of observations. $\hat{\lambda}_{i}$ denotes the i-th largest squared sample canonical correlation between $\Delta y_{t}$ and the respective cointegrating relation, both corrected for the influence of the remaining regressors. Since the trace test is known to be distorted in small samples, I implement a correction of the test statistic based on Cheung and Lai (1993). Critical values are obtained by computing the response surfaces in Doornik (1998), or Trenkler (2004) in case of breaks. Given the cointegration results, further LR tests are applied to check the null hypotheses of various parameter restrictions against the unrestricted model.

\subsection{Cycle Analysis}

Besides cointegration as rank reduction in the long-run multiplier matrix, I concentrate on short- and medium-term business cycle synchrony implying reduced rank in the short-run dynamics. More precisely, this means looking for a linear combination of the differenced time series, which is not predictable from the relevant past, thus eliminates all systematic autocorrelation structure.

Engle and Kozicki (1993) introduced the common cycles feature, which was taken up by Vahid and Engle (1993). They define a cycle as common, if some linear combination of the autocorrelated series is not serially correlated itself. This implies that there exists an $n \times s$ matrix $\gamma$ pre-multiplied in equation (3), such that $\gamma^{\prime} \alpha=0$ and $\gamma^{\prime} A_{i}=0$ for $i=1, \ldots, q$. That is, if $\gamma^{\prime}$ lies in the intersection of the left nullspaces of the longand short-run adjustment coefficients, one gets the innovation process $\gamma^{\prime} \Delta y_{t}=\gamma^{\prime} u_{t}$. Integration of both sides clarifies that $\gamma^{\prime}$ removes as well the cyclical component in the levels $y_{t}$, leaving a random walk with naturally uncorrelated innovations. It is obviously not worth considering VECMs with $q=0$, because $\gamma$ would simply be the orthogonal complement of $\alpha$.

Testing the null hypothesis of at most $s$ common cycle vectors is done by canonical 
correlation analysis between $\Delta y_{t}$ and the regressors from $(3), \Delta y_{t-i}(i=1, \ldots, q)$ and $\hat{\beta}^{\prime} y_{t-1}$. Note that $s$ cannot exceed $n-r$ (Vahid and Engle 1993), because the cofeature matrix must lie in the space of the $n \times(n-r)$ orthogonal complement of $\alpha$. The canonical correlations are conditioned on a constant and outlier neutralisation dummies. With the eigenvalues $\eta_{i}$ (in ascending order), the LR test statistic for the null hypothesis of at least $s$ common cycle vectors

$$
C C(s)=-(T-n(q-1)-r) \sum_{i=1}^{s} \log \left(1-\hat{\eta}_{i}\right)
$$

is calculated. The relevant $\chi^{2}$-distribution has $s(r+n q)-s(n-s)$ degrees of freedom. This can be seen, when applying the common cycle restrictions to the VECM from (3):

$$
\left(\begin{array}{cc}
I_{s} & \tilde{\gamma}^{\prime} \\
0_{(n-s) \times s} & I_{n-s}
\end{array}\right) \Delta y_{t}=\left(\begin{array}{c}
0_{s \times r} \\
\tilde{\alpha}
\end{array}\right)\left(\beta^{\prime} y_{t-1}+c_{0}+c_{1}(t-1)+c_{2} b_{t-1}\right)+c_{3} d_{t}+\sum_{i=1}^{q}\left(\begin{array}{c}
0_{s \times n} \\
\tilde{A}_{i}
\end{array}\right) \Delta y_{t-i}+v_{t} .
$$

The cofeature matrix has been normalised such that $\gamma^{\prime}=\left(I_{s}: \tilde{\gamma}^{\prime}\right)$. $\tilde{\alpha}$ and the $\tilde{A}_{i}$ contain the lower $n-s$ rows of $\alpha$ and the $A_{i}$; the upper rows are filled by $s \cdot r$ zero restrictions on $\alpha$ and $s \cdot n$ on each of the $q A_{i}$. The efficient estimation of the cofeature matrix requires $s \cdot(n-s)$ additional coefficients, leaving a parameter difference equal to the above-mentioned number of degrees of freedom. From (6) it is seen that the transitory movements of $s$ differences are absorbed in the cofeature relations. This implies that the $s$ smallest canonical correlations should be tested insignificant by (5).

While the common cycles concept calls for linear combinations not predictable at all, one could reduce this requirement to the short-run, meaning high frequencies. This leads to allowing for different factors generating the long- and short-term dynamics as in Hecq et al. (2006). In detail, the low-frequency fluctuations from the adjustment processes are considered as idiosyncratic. Therefore, in the search for unpredictable linear combinations, one corrects for the cointegration equilibrium effects: $\gamma^{\prime}\left(\Delta y_{t}-\alpha \beta^{\prime} y_{t-1}\right)=\gamma^{\prime} u_{t}$. Besides increasing flexibility, the high-frequency variant has the advantage of limiting the number of common cycle vectors only to $n-1$ instead of $n-r$. The appropriate model differs from (6) only in that $\alpha$ is left unrestricted. Accordingly, the test statistic is the same as in (5) with $r$ set to zero. The eigenvalues are estimated in the same canonical correlation programme, though now additionally conditioned on the error correction terms. 


\subsection{Permanent-Transitory Decomposition}

Until this point, I have presented tests for the number of common trends and common cycles among several time series. These numbers determined, the trending and cyclical components can be filtered out of the data. In this, I follow Proietti (1997), who provides the permanent-transitory decomposition, and Hecq et al. (2000), who add the common cycles feature.

First, define the matrix

$$
P=\left(A(1)-\alpha \beta^{\prime}\right)^{-1} \alpha\left(\beta^{\prime}\left(A(1)-\alpha \beta^{\prime}\right)^{-1} \alpha\right)^{-1} \beta^{\prime},
$$

where $A(L)=I_{n}-\sum_{i=1}^{q} A_{i} L^{i}$. Then, the common trends are given by

$$
(I-P)\left(A(1)-\alpha \beta^{\prime}\right)^{-1} \alpha_{\perp}\left(\alpha_{\perp}^{\prime} \alpha_{\perp}\right)^{-1} \alpha_{\perp}^{\prime} A(L) y_{t}
$$

with $\perp$ denoting the orthogonal complement (thus $\alpha^{\prime} \alpha_{\perp}=0$, where both $\alpha$ and $\alpha_{\perp}$ have full column rank). The common cycles result as

$$
\tilde{\gamma}_{\perp}\left(\tilde{\gamma}_{\perp}^{\prime} \tilde{\gamma}_{\perp}\right)^{-1} \tilde{\gamma}_{\perp}^{\prime}\left(P y_{t}-(I-P)\left(A(1)-\alpha \beta^{\prime}\right)^{-1} \tilde{A}(L) \Delta y_{t}\right)
$$

where $\tilde{A}(L)=\sum_{i=0}^{q-1} \tilde{A}_{i} L^{i}$ and $\tilde{A}_{i}=\sum_{j=i+1}^{q} A_{j}, i=0, \ldots, q-1$. Note that in case of highfrequency common cycles, expression (9) does not contain the transitory components corresponding to the equilibrium adjustment processes, which remain idiosyncratic.

\section{Empirical Evidence}

\subsection{Data}

This study employs GDPs of the Asian Tigers South Korea ("Korea" in the following), Singapore and Taiwan as standard measures of aggregated economic development. The fourth tiger, Hong Kong, is not included in the final model versions for reasons explained towards the end of the paper. Further South-East Asian countries like Malaysia or Thailand would be worth considering, however limited availability of quarterly GDP series of sufficient length and quality would introduce the usual severe caveats of multivariate 
time series analysis; nevertheless, an examination covering a larger set of Asian Pacific countries may be found in Weber (2006). Here, the scope is broadened by the USA as the world economic leader and Japan as the forerunner in the Asian industrialisation representing foreign influences. Quarterly data are taken out of the EcoWin databases for the sample 1975:1-2006:4, Japan starting only in 1980:1.

Because of inflation, different currencies and price levels as well as different population sizes, the raw GDP series are no adequate data. Accordingly, the series have been transformed as follows: Per capita GDP has been calculated dividing by total population, which was linearly interpolated to gain quarterly values. The nominal data have been deflated to the year 2000 level using the GDP price deflator. Finally, the year 2000 purchasing power parity conversion factors from the international comparison program of the World Bank have been employed to transform all series into US dollar. ${ }^{2}$ The calculated variables can be interpreted as per quarter amount of dollars one would have needed in the USA in 2000, to reach the same living standard as the foreigner in the respective country and period.

The Census X-12 seasonally adjusted GDPs are displayed in Figure 1. A first graphical inspection brings the following stylised facts to the fore: The South-East Asian countries seem to share a regionally balanced growth path, multiplying their GDPs within the last decades. The temporary halting of this successful development process due to the 1997/98 financial crisis is clearly visible in the series of Korea and Singapore, countries known for having struggled the most at the time. The subsequent upswing was interrupted by the world economic recession, which can as well be seen in the US series. In Japan, the economic boom in the late 1980s is traceable just as the long period of deflationary recession. In this decade of low growth, the South-East Asian countries were able to catch up with Japan, while convergence with the US was far more gradual.

Before digging into the formal model analysis, the unit root properties of the time series are explored. In Table 1 the results of the ADF tests, respectively the Saikkonen and Lütkepohl (2002) tests for the breaking series, are presented. As mentioned above, shifts in 1998:1 are considered for Korea and Singapore in order to protect the analysis against distortion through crisis effects. Since no evidence against the null hypotheses can be found and the first differences are clearly stationary, I assume all examined GDPs integrated of order one. The calculations in this paper have been performed using EViews 5.0, JMulti 4.14, R 1.4 and Gauss 8.0.

\footnotetext{
${ }^{2}$ For Taiwan, the factor has been calculated by a PPP update based on the 1990 relative price from Penn World Table.
} 

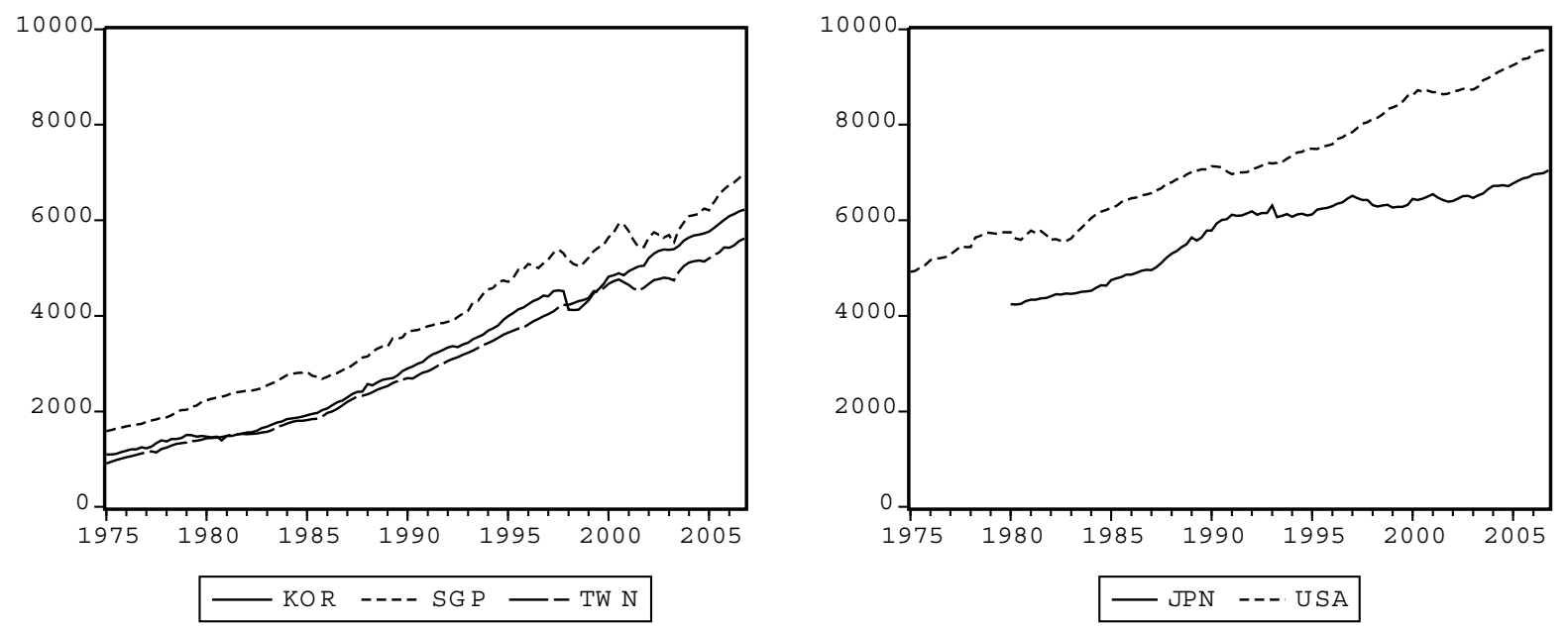

Figure 1: Real GDPs (2000 per capita purchasing power US $\$$ )

\begin{tabular}{|l|c|c|c|c|c|}
\hline & KOR & SGP & TWN & JPN & USA \\
\hline t-value & -0.87 & -2.50 & -2.16 & -1.47 & -2.41 \\
\hline break date & $1998: 1$ & $1998: 1$ & - & - & - \\
\hline lag length & 3 & 6 & 6 & 3 & 2 \\
\hline \multicolumn{6}{|c|}{ cannot be rejected at 10\% significance level } \\
constant and trend included \\
\hline
\end{tabular}

Table 1: Unit root tests on GDPs

\subsection{Model Specification}

Convergence among the South-East Asian countries of Korea, Singapore and Taiwan is analysed within the trivariate VECM from (3). Before addressing econometric results and economic implications, I discuss the model specification: While in the deterministic part, constant and trend are included, any level breaks $b$, above all at the time around the financial crisis, remained insignificant and proved irrelevant for the long-run model properties. Even though such permanent shifts were dispensable, three impulse dummies $d$ neutralise extreme residual outliers: 1988:1 might be associated to a short-time growth peak following the slow-down after the "Black Monday", in 1998:1 the financial crisis effect mostly in the Korean series is accommodated, and 2003:3 corresponds to an exceptional quarter in the Singaporean and Taiwanese recovery process after the world economic downturn. The Hannan-Quinn and Schwarz Criteria preferred a short lag length of only one quarter. Since such a specification left considerable autocorrelation in the residuals, I follow the Akaike Criterion, which suggests setting $q=6$.

The trace test statistic (4) is employed to uncover the number of cointegrating relations 
$r$ among the three series. $H_{0}: r=0$ is rejected on the $1 \%$ level $(\Lambda(0)=53.73$, critical value $=48.87)$, and since $\Lambda(1)=26.17$ still exceeds the $5 \%$ critical value of 25.73 , there is even evidence against $H_{0}: r=1$. Therefore, I allow for two cointegrating relations, both of which are found clearly stationary when applying ADF tests. This result does not depend on the particular deterministic specification and sample choice, but is not robust against substantially reducing the lag length. However, as mentioned above, a small $q$ does not appropriately capture the serial dynamics, supporting the sensibility of the current model set-up. This is underlined by Table 2, showing that the LM hypotheses of no autocorrelation up to the respective lag and the Jarque-Bera hypothesis of normality cannot be rejected.

\begin{tabular}{|c|c|c|c|c|}
\hline $\operatorname{LM}(1)$ & $\operatorname{LM}(2)$ & $\operatorname{LM}(4)$ & $\operatorname{LM}(8)$ & JB \\
\hline 0.56 & 0.77 & 0.60 & 0.10 & 0.47 \\
\hline
\end{tabular}

Table 2: Specification tests: p-values

\subsection{Cointegration and Convergence}

The trace test results meet the Bernard and Durlauf requirement of pair wise cointegration, implying one common stochastic trend in the GDPs of all three countries. Therefore, seen from a growth perspective, the driving forces of economic development processes are shared in the South-East Asian region. As argued in section 2, this strong result can arise from common factors as well as interaction within the region. Both potential sources will be assessed as the analysis proceeds. However, the convergence definition is not limited to cointegration, but comprises equal weights in the long-run relations; otherwise, the strength of the stochastic trend would differ across nations, reflecting non-aligned growth paths.

Consequently, I test the hypothesis that the row vectors in $\beta^{\prime}$ are equal to $(1,0,-1)$ and $(0,1,-1)$, where the variables KOR, SGP and TWN are simply in alphabetical order. An LR test produces a p-value of 0.37 (test statistic $=1.98$ with two degrees of freedom), so that the restrictions cannot be rejected. The error correction part of model (3) takes the form 


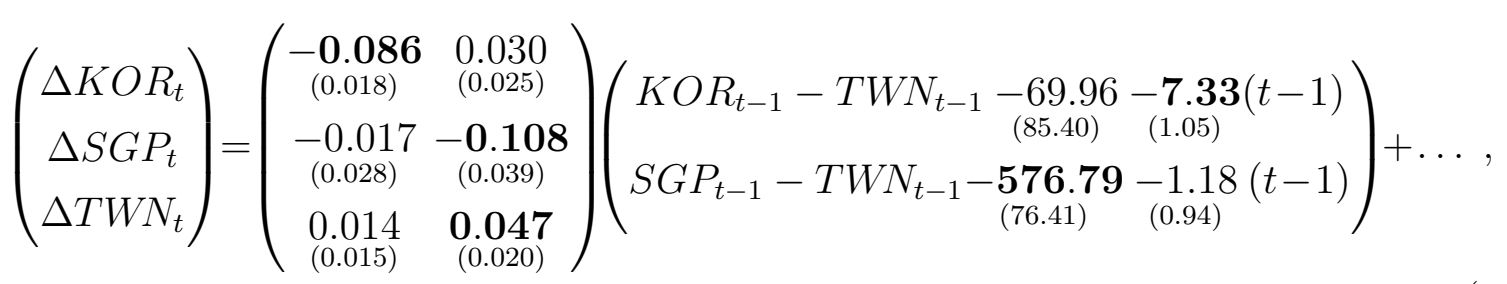

with standard errors in parentheses and significant estimates in bold. The deterministics reveal a significant initial gap between the Singaporean and Taiwanese GDPs, as can already be seen from Figure 1. The significant linear trend in favour of the Korean compared to the Taiwanese development is mostly owed to the sustained influence of the 2001 recession and the delayed crisis impacts in Taiwan. In general, since magnitudes are rather small and simple extrapolations into the future are surely not appropriate, one should not put too much weight on the deterministic model part.

Concerning the adjustment processes, the finding is that of interdependence in the long run: Deviations from the growth equilibrium affect the further GDP development of all countries. These adjust in the "correct" direction, thereby re-equilibrating the system as time passes by. The smallest coefficients show up in the equation of Taiwan, which thus maintains the highest degree of independence. In contrast, the small economy of Singapore reveals the strongest reactions; significantly, this size effect will reappear below.

\subsection{Business Cycle Coherence}

Until now, the stochastic trend analysis has been subject of interest. Laying the focus on medium-run fluctuations, this section presents results on common cycles as the second conceptual element. The tests for common cycles are conducted as described in section 3.3. Since two cointegrating relations have been detected, $n-r=1$ is an upper bound for the number of common cycle vectors. For the according hypothesis $H_{0}: s \geq 1$ the p-value equals 0.15 (test statistic $=24.10$ with 18 degrees of freedom), so that the presence of one common cycle vector can be accepted.

By the high-frequency variant, it is possible to test for the maximum number of two common cycle vectors. Since a positive result would indicate the presence of a single (high-frequency) cyclical component shared by all countries involved, this task is of special interest. With a p-value of 0.48 , the hypothesis $H_{0}: s \geq 1$ (test statistic $=15.56$ with 
16 degrees of freedom) cannot be rejected. ${ }^{3}$ Even for $H_{0}: s \geq 2$, the p-value still comes to 0.18 (test statistic $=41.45$ with 34 degrees of freedom), allowing the adoption of two common cycle vectors. Since both test variants justify adopting the minimal statistically attainable number of common transitory components, they provide evidence for close connections in the cyclical fluctuations of the different GDPs.

The restriction of one high-frequency common cycle can now be applied to the VECM from (3), in order to estimate the cofeature coefficients with standard errors. The cofeature matrix results as

$$
\gamma^{\prime}=\left(\begin{array}{ccc}
1 & 0 & -0.540 \\
& & (0.125) \\
0 & 1 & -1.571 \\
& & (0.218)
\end{array}\right)
$$

For instance, the estimates imply that the transitory fluctuations of Korea (the first variable) on the right hand side of (6) can be eliminated by subtracting 0.540 times the Taiwanese variable (third). An estimate of -1 would indicate coherent cycles of equal per capita strength. Therefore, the common cycle is strongest in the Singaporean GDP, followed by Taiwan and Korea. The clear significance of the parameters supports the decision of introducing common feature restrictions.

The presence of a common business cycle imposes the question of transmission effects between the countries involved. For the high-frequency cycle, this means looking for spillover interaction that appears apart from the long-run growth effects. A logical procedure thus consists of testing Granger non-causality restrictions on the short-run dynamics in the VECM from (3). For this purpose, I define the hypotheses that each respective country is not subject to any short-run influences from the remaining two, or formally, that both off-diagonal elements in the respective row of all $A_{i}(i=1, \ldots, 6)$ equal zero. Table 3 displays the LR test statistics with 12 degrees of freedom.

\begin{tabular}{|c|c|c|}
\hline$H_{0}: S G P, T W N \not \rightarrow K O R$ & $H_{0}: K O R, T W N \not \mapsto S G P$ & $H_{0}: K O R, S G P \not \rightarrow T W N$ \\
\hline 20.47 & $51.76^{* *}$ & $27.62^{*}$ \\
\hline \multicolumn{2}{|c|}{$* *, *: H_{0}$ can be rejected at $5 \%$ respectively $1 \%$ significance level } \\
\hline
\end{tabular}

Table 3: LR tests on short-run Granger non-causality

Evidence against the hypothesis of no influence received is highest for Singapore, followed by Taiwan and Korea. Again, an appealing interpretation can therefore link the degree of

\footnotetext{
${ }^{3}$ Remember that $s$ now denotes the number of high-frequency common cycle vectors.
} 
independence from cyclical spillovers to the size of the economies. Note as well that the same order of countries was found concerning the strength of the business cycle. Naturally, the outcome of Granger causality tests depends on the set of variables included; while the system restricted to South-East Asian economies already provided interesting insights, the roles of Japan and the US as world factors will be explored in section 4.6.

\subsection{Decomposing GDPs}

The preceding sections have dealt with examining the strength of economic integration. Thereby, high coherence could be established within South-East Asia concerning long-run growth paths as well as business cycle fluctuations. In the following, I filter out both common trend and common cycle, in order to analyse outward economic linkages of the region as a whole.

The common trend is calculated as in (8), where all loadings are equal due to the restrictions on the cointegrating vector. The deterministic parts are handled as follows: First, the derived common trend is subtracted from each GDP series. The three rest components are then regressed on a constant and a linear trend. The mean constant and mean linear trend from these regressions are finally added to the common stochastic trend. This procedure takes the deterministics in the estimated VECM into account and provides a sensible measure of the regional growth path. The common cycle series is constructed from (9). In equivalence to the stochastic trend, constant and linear trend from an OLS regression are subtracted from the cycle. Figure 2 plots the obtained series.
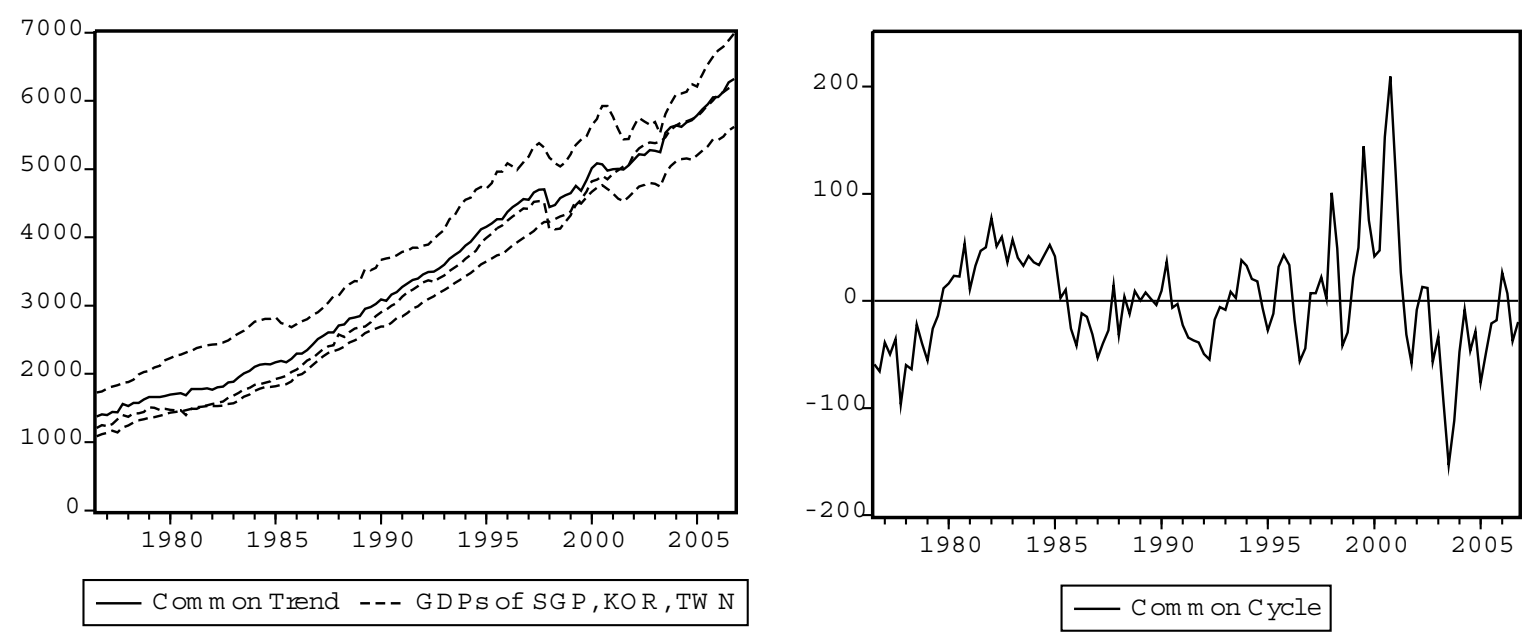

Figure 2: Common South-East Asian trend and high-frequency cycle

Due to the calculation of the deterministics, the common stochastic trend lies midst of 
the underlying GDP series. In the cyclical pattern, one can detect an upswing in the early 1980s ended by the mid-80s economic crisis, a trough corresponding to the world recession in the early 1990s, the enormous recovery process after the Asian financial crisis and the hefty downturn in the first recession following the millennium. The crisis in 1998 is mainly picked up by the trend component, suggesting a permanent effect on the income level.

The GDPs of Japan and the US are decomposed in comparable univariate procedures: At first, the ARIMA models

$$
\begin{gathered}
\Delta J P N_{t}=\underset{(6.861)}{26.356}+\underset{(0.097)}{0.214 \Delta J P N_{t-3}+\hat{v}_{1 t} \quad \text { and }} \\
\Delta U S A_{t}=\underset{(6.821)}{36.454}+\underset{(0.089)}{0.231 \Delta U S A_{t-1}}+\underset{(0.089)}{0.159 \Delta U S A_{t-2}}+\hat{v}_{2 t}
\end{gathered}
$$

are estimated. Thereby, the specifications based on information criteria and parameter significance are checked by testing for absence of residual autocorrelation. Then, the obtained residuals $\hat{v}$ are sequentially accumulated to random walk series. After the deterministics have been subtracted and added as above, at last the cycle is defined as the difference between series and trend. Other sensible ARIMA settings leading to results substantially different from the following could not be found.

\subsection{South-East Asia in the World Economy}

Since all permanent and transitory components are derived, this section can address international linkages of the South-East Asian region. Starting with business cycle connections, for reasons of clarity the analysis is focused on the major cases of Japan and the US. In a first step, I consider the degree of alignment between the transitory fluctuations. For this purpose, Figure 3 (left panel) presents rolling five-year correlations between the South-East Asian and the respective foreign cycle. The window size seems appropriate for business cycle horizons, since shorter time spans merely produce volatile and hardly interpretable correlations, while larger ones are not likely to catch the development of coherence over time.

As a first impression, correlations with the US cycle are higher than with Japan. Nonetheless, in the beginning, they are significantly negative; in the early 1980s, the South-East Asian economy was still booming, while the US already went through a recession. After the South-East Asian downturn in the mid-1980s crisis, significantly positive correlations 

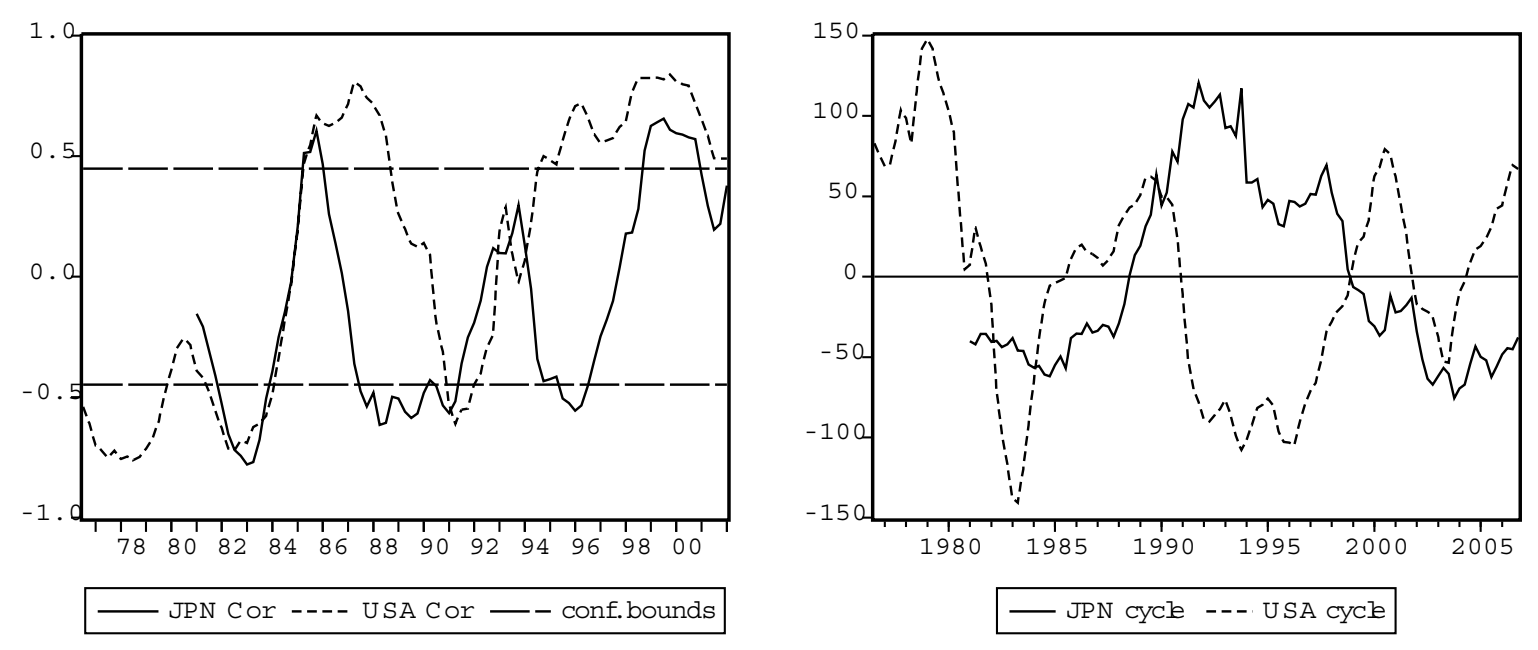

Figure 3: Correlations between South-East Asian and foreign cycles (see right panel)

with the US appear for several time windows comprising the late 1980s and early 1990s. The following recession had a much higher impact in the US than in South-East Asia, thereby re-diminishing correlations. Since the mid-1990s though, values stabilise at about 0.5 due to the fact, that millennium boom, recession and the latest upswing are shared by South-East Asia and the US. The Japanese cycle mirrors both the bubble economy and the following deflationary stagnation, resulting in an unusual "cyclical" pattern. The fluctuating correlations confirm that South-East Asia did not follow this idiosyncratic course. Only in the last years, when the Japanese situation had normalised, significant positive correlations appear, but do not reach the US level.

Correlation of cycles might be due to influences in one or both directions, but can as well appear in presence of common shocks. Granger causality analysis is likely to provide further information on this topic. For this reason, in analogy to Figure 3, I estimate fiveyear rolling VAR models including the South-East Asian and one of the foreign cycles, respectively. A lag length of two proved a sensible choice, which is not decisive for the qualitative results. LR tests of zero restrictions on the respective off-diagonal parameters are performed. This corresponds to the hypotheses in the legends of Figure 4, which presents the rolling LR test statistics from both models.

The left panel clarifies that systematic influences between the South-East Asian and the Japanese business cycles do not exist. This result is not due to low test power in the short time windows, since longer estimation periods neither allow to reject the null hypotheses. In contrast, evidence for US influences on the South-East Asian cycle can be found in the right panel. These are however restricted to the periods of high correlations, 

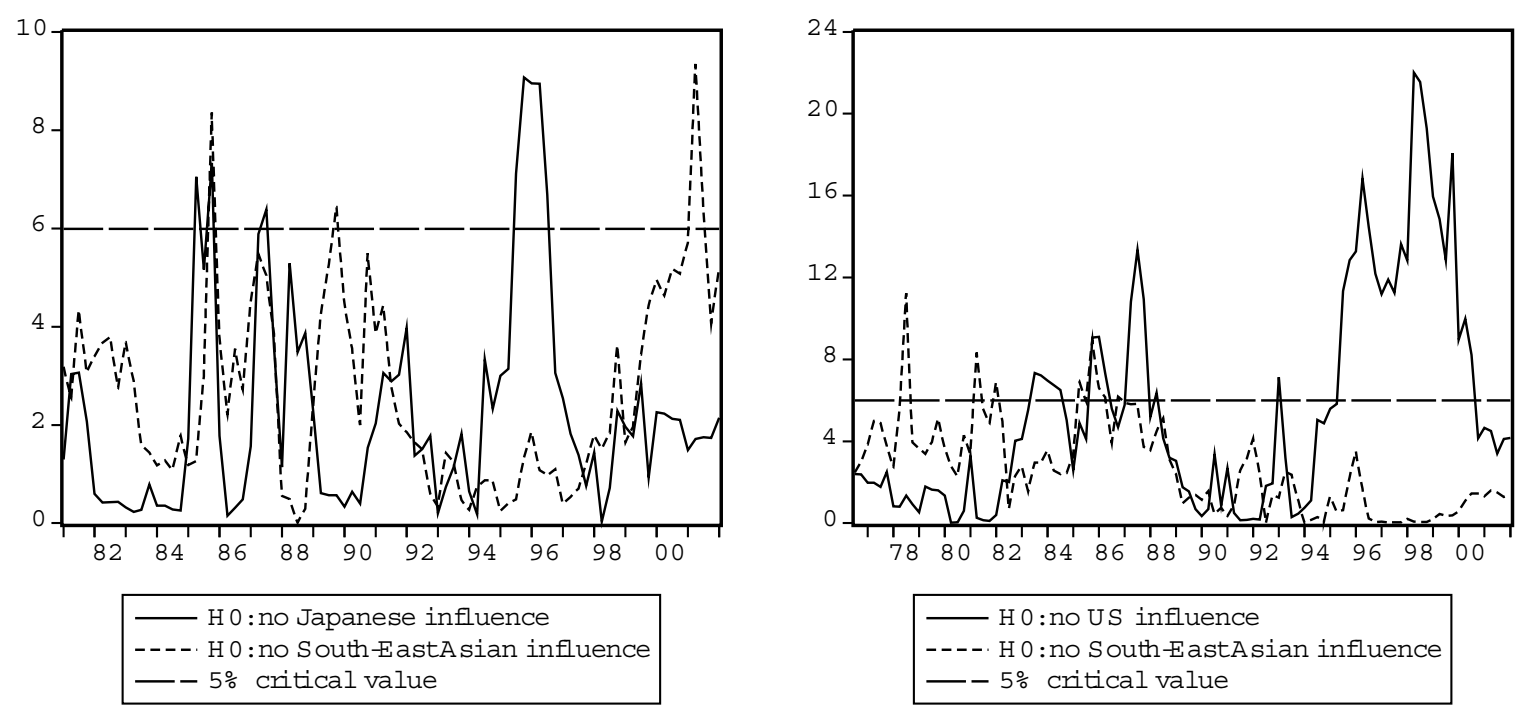

Figure 4: LR causality tests between South-East Asian and foreign cycles

which had been identified above. Nonetheless, it can be stated that cyclical coherence is a result of single-direction US impacts, while Japanese spillovers are not apparent. Obviously, despite the regional proximity, Japan cannot match with the super power US in originating major business cycle impulses.

The second point of interest is the stance of the South-East Asian region in international growth and convergence processes. Again, this issue is addressed following the Bernard and Durlauf concept, which suggests testing for cointegration between the common SouthEast Asian trend and the permanent components of Japan and the US. Since rolling windows normally provide too few observations for this type of analysis, the trace test statistics in Figure 5 are estimated backward recursively, meaning samples with fixed end and moving starting point. By construction of the stochastic trend series, the lag length $q$ is zero, and constant and linear trend have been included as usual.

For Japan, the null hypothesis of no cointegration can be rejected from 1993:1 onwards. The US statistic reveals far less significance, but reaches the critical value in 1998:1. Even as this date seems to ascribe a decisive effect to the Asian financial crisis, neither including a structural break nor cutting the sample in 1997:4 can reveal cointegration in earlier periods. That is, data is consistent with long-run linkages between South-East Asia and the two matured industrial nations emerging in the last one or two decades. Problems of time series tests in finding convergence can thus be attributed to dynamic adjustments of the structural growth properties through time; this finding corresponds to the protectionist and regulatory course of the Tigers after World War II, which gave way 


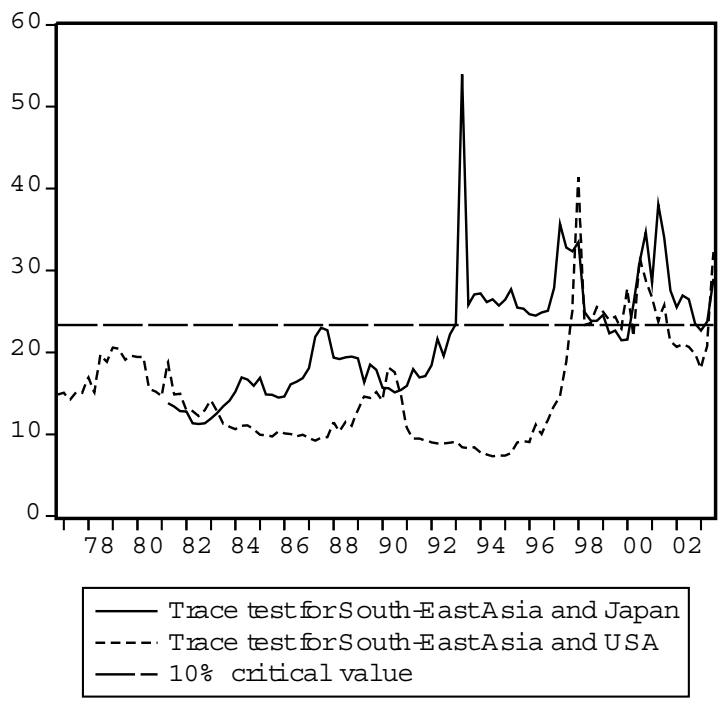

Figure 5: Trace tests between South-East Asian and foreign trends

to market- and trade-oriented strategies later on.

In the VECMs, which are estimated for the time spans indicated by the earliest significant trace tests, the hypothesis $\beta^{\prime}=(1,-1)$ can be checked. For Japan, the LR p-value is 0.61 (test statistic $=0.27$ with one degree of freedom), for the US it reaches 0.85 (test statistic $=0.03$ with one degree of freedom). Therefore, both restrictions are clearly accepted, leading to the following systems (with SEA denoting South-East Asia):

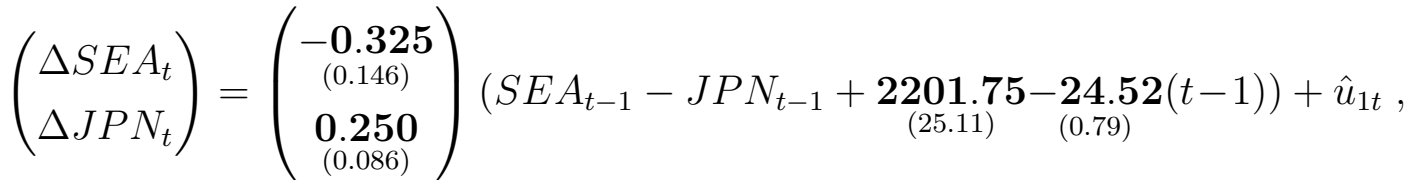

$$
\begin{aligned}
& \left(\begin{array}{c}
\Delta S E A_{t} \\
\Delta U S A_{t}
\end{array}\right)=\left(\begin{array}{c}
-\mathbf{0 . 7 2 8} \\
(0.173) \\
0.085 \\
(0.119)
\end{array}\right)\left(S E A_{t-1}-U S A_{t-1}+\underset{(23.82)}{\mathbf{3 7 3 7 . 4 7}} \underset{(1.15)}{\mathbf{1 2 . 6 3}(t-1)}\right)+\hat{u}_{2 t} .
\end{aligned}
$$

In the deterministics, the significant constants mirror the considerable initial lead of Japan and even more the US over South-East Asia. However, this gap is gradually reduced, as can be seen from the significant linear trends. Thereby, the speed of catching-up towards Japan (24.52 per capita PPP US \$ per quarter) doubles the one towards the US (12.63), a consequence of the Japanese "lost decade". I sum, all the convergence prerequisites, cointegration, equal weights and catching-up, are fulfilled.

From the adjustment vectors it can be seen that long-run interdependence prevails between South-East Asia and Japan, even if the higher influence naturally comes from the 
latter. Contrastingly and in line with a priori expectations, the US represents a weakly exogenous variable, inducing substantial adjustment of South-East Asia. Including all three GDP trends in a common system confirms this constellation: The far largest proportion of long-run variation in South-East Asian growth turns out to be governed by US shocks.

Finally, it might be worthwhile shortly considering the special role of the fourth tiger Hong Kong: Cointegration tests and VECM estimations remained unclear through several specifications, detailed results are left out to save space. When included in the South-East Asian system, adjustment of Hong Kong to the cointegrating relations either did not reach significance, or proved even wrong-directed, thereby enlarging occurring equilibrium deviations. Since stability of the system is nonetheless guaranteed by the neighbour countries, this constellation could possibly be interpreted as favouring a regional leading role of Hong Kong. While this may be explained by extreme openness in goods and financial markets, tight contacts to the US and China, and extremely fast recovery processes in the aftermath of economic crises, further research is required to substantiate a consistent argumentation.

\section{Concluding Summary}

In this paper, I have focused on stance and properties of the macroeconomic integration in the South-East Asian region. Stochastic convergence, business cycle coherence as well as intraregional and foreign impulse transmission were the main points of interest. In summarising the empirical results, I will conclude by closing the loop to the questions set out in the introduction.

The cointegration analysis has been motivated by a convergence criterion arising from neoclassical growth theory, which requires a cointegrating long-run equilibrium with equal weights on different per capita GDPs. In fact, evidence is in favour of common stochastic trends in the GDPs of South Korea, Singapore and Taiwan, which convincingly meet the convergence conditions. As the second element in the integration concept, I examined the synchrony of business cycle fluctuations. In this, a cycle is defined as common, if it is possible to cancel out all autocorrelation dynamics in a linear combination of the GDPs. Applying a high-frequency common cycles test, the null hypothesis of a single common serial correlation feature could not be rejected. Spillovers of growth shocks emerge in the domains of both the long and the short run and are consistent with the different size of 
the economies.

It turns out that in terms of fundamental macro developments, the three countries form a homogenous economic region. Important implications relate to various topics: First, openness in goods and financial markets has brought all countries on an obviously successful growth path. Future trade arrangements and FDI policies can surely take advantage of this fact. Furthermore, the high degree of homogeneity is likely to facilitate regional monetary cooperation, because similar business cycles and structural growth determinants prevent the problem of inappropriate common policies in presence of diverging national developments. Thereby, the task of exchange rate arrangements is of particular importance in view of Asian structural vulnerability, which might be reduced in a more robust and credible currency bloc. In general, regional institutionalisation is straightforwardly tackled both as outcome of and as prerequisite for a sound integrated development.

Based on the test results, the common stochastic trend and cycle were extracted from the data, representing the South-East Asian region in a broader analysis: In detail, the focus is on examining the relations towards the leading economies of Japan and the US. Periodical correlations of the business cycles were found only with the US, which prove causal for the South-East Asian fluctuations. Long-run convergence towards Japan can be confirmed since the early 1990s and towards the US since the late 1990s. While the latter again comes out as an exogenous driving force of growth processes, mutuality characterises the connections to Japan.

These pieces of evidence raise two central issues: On the one hand, the newly industrialised South-East Asian countries profoundly head towards full integration into the group of developed economies, thereby setting an example of successful growth strategies. On the other hand, substantial dependence on influences from abroad should be taken into account. Such a situation bears the potential of permanently initialising further progress, but latent threats in a globalising world are to be treated with caution. Future research might explore the Chinese role in the interrelations of the new Asian growth dynamics, while coping with the well-known substantial data problems.

\section{References}

[1] Bayoumi, T., B. Eichengreen (1994): One Money or Many? Analyzing the Prospects of Monetary Unification in Various Parts of the World. Princeton Studies in International Finance, 76. 
[2] Bernard, A.B., S.N. Durlauf (1995): Convergence in international output. Journal of Applied Econometrics, 10, 97-108.

[3] Bernard, A.B., S.N. Durlauf (1996): Interpreting tests of the convergence hypotheses. Journal of Econometrics, 71, 161-173.

[4] Canova, F., H. Dellas (1993): Trade interdependence and the international business cycle. Journal of International Economics, 34, 23-47.

[5] Cheung, Y.W., K.S. Lai (1993): Finite sample size of Johansen's likelihood ratio tests for cointegration. Oxford Bulletin of Economics and Statistics, 55, 313-328.

[6] Davidson, R., J. MacKinnon (1993): Estimation and Inference in Econometrics. Oxford University Press, London.

[7] Doornik, J.A. (1998): Approximations to the asymptotic distributions of cointegration tests. Journal of Economic Surveys, 12, 573-593.

[8] Engelbrecht, H.-J., B. Kelsen (1999): Economic Growth and Convergence Amongst the APEC Economies 1965-1990. Asian Economic Journal, 13, 1-17.

[9] Engle, R.F., S. Kozicki (1993): Testing for Common Features (with comments). Journal of Business and Economic Statistics, 11, 369-395.

[10] Hecq, A., F.C. Palm, J.-P. Urbain (2000): Permanent-Transitory Decomposition in VAR Models with Cointegration and Common Cycles. Oxford Bulletin of Economics and Statistics, 62, 511-532.

[11] Hecq, A., F.C. Palm, J.-P. Urbain (2006): Common Cyclical Features Analysis in VAR Models with Cointegration. Journal of Econometrics, 132, 117-141.

[12] Jansen, W.J., A.C.J. Stokman (2004): Foreign direct investment and international business cycle comovement. ECB Working Paper Series, 401, European Central Bank.

[13] Johansen, S. (1994): The role of the constant and linear terms in cointegration analysis of nonstationary time series. Econometric Reviews, 13, 205-231.

[14] Johansen, S. (1995): Likelihood-based Inference in Cointegrated Vector Autoregressive Models. Oxford University Press, Oxford.

[15] Johansen, S., R. Mosconi, B. Nielsen (2000): Cointegration analysis in the presence of structural breaks in the deterministic trend. Econometrics Journal, 3, 216-249. 
[16] Lanne, M., H. Lütkepohl, P. Saikkonen (2002): Comparison of unit root tests for time series with level shifts. Journal of time series analysis, 23, 667-685.

[17] Lee, H.A., K.P. Lim, V.K.S. Liew (2004): Income convergence between Japan and the rest of East Asian countries. Journal of International Business and Economics, 1, 63-71.

[18] Perron, P. (1989): The great crash, the oil price shock and the unit root hypothesis. Econometrica, 57, 1361-401.

[19] Proietti, T. (1997): Short-Run Dynamics in Cointegrated Systems. Oxford Bulletin of Economics and Statistics, 59, 405-422.

[20] Saikkonen, P., H. Lütkepohl (2002): Testing for a unit root in a time series with a level shift at unknown time. Econometric Theory, 18, 313-348.

[21] Trenkler, C. (2004): Determining p-values for systems cointegration tests with a prior adjustment for deterministic terms. CASE Discussion Paper, 37, HumboldtUniversität zu Berlin.

[22] Vahid, F., R.F. Engle (1993): Common Trends and Common Cycles. Journal of Applied Econometrics, 8, 341-360.

[23] Weber, E. (2006): Macroeconomic Integration in Asia Pacific: Common Stochastic Trends and Business Cycle Coherence. CRC 649 Discussion Paper 2006-039, Humboldt-Universität zu Berlin. 